\title{
A novel $K L F 13$ mutation underlying congenital patent ductus arteriosus and ventricular septal defect, as well as bicuspid aortic valve
}

\author{
PRADHAN ABHINAV ${ }^{1 *}$, GAO-FENG ZHANG ${ }^{2 *}$, CUI-MEI ZHAO ${ }^{3 *}$, \\ YING-JIA XU ${ }^{2}$, JUAN WANG ${ }^{1}$ and YI-QING YANG $^{2,4,5}$

\begin{abstract}
${ }^{1}$ Department of Cardiology, East Hospital, Tongji University School of Medicine, Shanghai 200120;
${ }^{2}$ Department of Cardiology, Shanghai Fifth People's Hospital, Fudan University, Shanghai 200240;

${ }^{4}$ Cardiovascular Research Laboratory; ${ }^{5}$ Central Laboratory, Shanghai Fifth People's Hospital,
\end{abstract} \\ ${ }^{3}$ Department of Cardiology, Tongji Hospital, Tongji University School of Medicine, Shanghai 200065; \\ Fudan University, Shanghai 200240, P.R. China
}

Received December 21, 2021; Accepted February 11, 2022

DOI: $10.3892 /$ etm.2022.11240

\begin{abstract}
Recently, mutations in the Kruppel-like factor 13 (KLF13) gene encoding a Kruppel-like transcription factor have been reported to cause congenital heart disease (CHD). However, due to pronounced genetic heterogeneity, the mutational spectrum of KLF13 in other cohorts of cases suffering from distinct types of CHD remain to be ascertained. In the present investigation, by Sanger sequencing of KLF13 in 316 unrelated cases affected by different forms of CHD, a new mutation in heterozygous status, NM_015995.3: c.430G $>$ T; p.(Glu144*), was detected in an index patient affected with patent ductus arteriosus (PDA) and ventricular septal defect (VSD), as well as bicuspid aortic valve (BAV), with a mutation frequency of $\sim 0.32 \%$. Genetic investigation of the available family members of the proband demonstrated that the truncating mutation co-segregated with CHD. The nonsense mutation was not observed in 400 unrelated volunteers without CHD who were enrolled as control subjects. Quantitative biological measurements with dual luciferase reporters revealed that Glu144*-mutant KLF13 did not transactivate the downstream genes vascular endothelial growth factor A and natriuretic peptide $\mathrm{A}$. In addition, the mutation abrogated
\end{abstract}

Correspondence to: Dr Juan Wang, Department of Cardiology, East Hospital, Tongji University School of Medicine, 150 Jimo Road, Shanghai 200120, P.R. China

E-mail:wj57188@163.com

Dr Yi-Qing Yang, Cardiovascular Research Laboratory, Shanghai Fifth People's Hospital, Fudan University, 801 Heqing Road, Shanghai 200240, P.R. China

E-mail: yangyiqing@fudan.edu.cn

*Contributed equally

Key words: congenital heart defect, molecular genetics, KLF13, transgene, biochemical assay the synergistic transcriptional activation between KLF13 and T-box transcription factor 5, a well-established CHD-causing gene. In conclusion, the present study indicates that genetically defective $K L F 13$ contributes to familial PDA and VSD, as well as BAV, which expands the phenotypic spectrum linked to $K L F 13$, and reveals a novel molecular pathogenesis of the disease, providing a new molecular target for the early prophylaxis and individualized treatment of CHD.

\section{Introduction}

Congenital heart disease (CHD), as a collective diagnosis for structural malformations of the heart and valves, as well as the endothoracic great blood vessels, occurring during embryonic development, represents the most common birth deformity in humans, with a prevalence of $\sim 1 \%$ among live births worldwide $(1,2)$. When minor cardiac structural anomalies are included, such as aneurysm of the atrial septum and bicuspid aortic valve (BAV), which is the most prevalent congenital cardiovascular anomaly with an incidence of 1-2\% in the population, the overall prevalence of CHD may be as high as $\sim 5 \%$ (2). Based on the occurrence of cardiac lesions in certain locations, CHD is clinically classified into $>20$ distinct subtypes, including ventricular septal defect (VSD), patent ductus arteriosus (PDA), transposition of the great arteries (TGA), double outlet of the right ventricle (DORV), tricuspid valve atresia (TVA), atrial septal defect (ASD), endocardial cushion defect, aortic stenosis, a right aortic arch, a single ventricle, tetralogy of Fallot, hypoplastic left heart and hypoplastic right heart (3-6). Irrespective of minor CHD that may resolve spontaneously (3), major CHD may contribute to diminished health-associated quality of life (7-9), decreased exercise performance (10-14), delayed neurodevelopment and brain injury (15-18), ischemic or hemorrhagic cerebral stroke (19-21), pulmonary arterial hypertension or Eisenmenger syndrome (22-24), viral pneumonia (25-27), infective endocarditis (28-30), acute myocardial infarction $(31,32)$, chronic congestive heart failure (33-35), ventricular or supraventricular 
arrhythmia (36-38) and death (39-42). Notably, CHD remains the most frequent etiology of newborn deaths caused by birth defects, with $21.8 \%$ of the neonates who succumb to various birth malformations having a cardiovascular abnormality (43). Although tremendous improvement in the outcomes of cardiac surgery and perioperative intensive care has been achieved, which allows $>90 \%$ of CHD-infants to survive into adulthood and reach fertile age, this results in an increasing adult population with CHD, and now the rising number of adult patients with CHD accounts for more than two-thirds of the overall CHD population (44). Moreover, the rates of late surgical complication and cardiac comorbidity, and even mortality, markedly increase in adults affected with CHD (45-47). Despite the clinical importance, the etiologies underpinning CHD in a considerable proportion of cases remain obscure.

In vertebrates, the heart is the first functional organ that develops during embryonic morphogenesis, and cardiac organogenesis undergoes an extremely complex biological process, which is precisely mediated by a sophisticated regulatory network, involving transcription factors, cardiac structural proteins, signaling transducers, epigenetic modifiers and microRNAs (48). Previous studies have demonstrated that both environmental risk factors and genetic defects may interfere with this finely controlled developmental process, giving rise to CHD (1,2,48-54). The well-recognized non-inheritable pathogenic factors for CHD include maternal viral infection, nutritional deficiency, obesity, diabetes and decreased physical activity, as well as exposure to toxic chemicals, therapeutic drugs and ionizing radiation during early pregnancy (48-51). However, accumulating evidence highlights the strong genetic basis underpinning CHD (1,2,52-54). Significant familial aggregation of CHD has been reported, with the risk of CHD recurrence in the first-degree offspring of an affected parent being between 3 and 19\% depending on the distinct types of lesion (55). In addition to chromosomal alterations encompassing aneuploidies, microdeletions and microduplications, pathogenetic variations in $>100$ genes amply expressed in the developing heart, encompassing those encoding sarcomeric proteins, transcription factors, chromatin modifies and signal-transducing molecules, have been determined to contribute to CHD $(1,2,52,53,56-83)$. Of these reported CHD-causative genes, the majority code for cardiac core transcription factors, such as T-box transcription factor (TBX)1, TBX20, TBX5, NK2 homeobox 5, GATA binding protein (GATA)6, GATA4, GATA5, heart and neural crest derivatives expressed (HAND)1 and HAND2 (84). However, the genetic components underpinning CHD in most cases are still unknown.

Notably, mutations in the Kruppel-like factor 13 (KLF13) gene, which codes for a Kruppel-like transcription factor crucial for proper cardiovascular morphogenesis, have recently been discovered to cause distinct types of CHD $(66,67)$. Li et al (66) performed targeted sequencing analyses of the entire coding region of KLF13 in a cohort of 309 index patients suffering from $\mathrm{CHD}$, and found two heterozygous $K L F 13$ variants in 2 out of 309 CHD patients, including NM_015995.3: c.467G >A; p.(Ser156Asn) in one patient affected with TGA and NM_015995.3: c.487C>T; p.(Pro163Ser) in another patient with TVA, VSD and ASD. These two missense mutations were absent from 200 population-matched healthy controls.
Biological assays elucidated that Ser156Asn-mutant KLF13 had enhanced transcriptional activation on the downstream target gene brain natriuretic peptide, alone or in synergy with TBX5, and a significantly enhanced ability to bind physically to TBX5, whereas the Pro163Ser variant showed a loss-of-function effect (66). Wang et al (67) performed whole-exome sequencing analyses in a family with a high incidence of CHD (DORV and VSD), and identified a new KLF13 variant, NM_015995.3: c.370G>T; p.(Glu124*). The nonsense heterozygous mutation was absent from 312 control individuals without CHD. Functional investigation unveiled that the Glu124* variation exerted a loss-of-function impact on its two target genes, actin $\alpha$ cardiac muscle 1 (ACTC1) and atrial natriuretic peptide, singly or synergistically with GATA4, as well as GATA6 (67). These investigations underscore the substantial genetic heterogeneity of CHD comprising a wide spectrum of cardiovascular structural malformations, which encourages exploration of the spectrum and prevalence of KLF13 variations in different cohorts of cases inflicted with various types of CHD. The aim of the current study was to analyze the spectrum and prevalence of $K L F 13$ variations in another cohort of cases with various types of CHD.

\section{Materials and methods}

Recruitment and clinical evaluation of study participants. The present study participants comprised a new cohort of 316 index patients affected with different forms of CHD enrolled from the Chinese Han population between March 2018 and November 2020 at Tongji Hospital and East Hospital, Tongji University (Shanghai, China). Clinical diagnosis and classification of various types of CHD were made as described previously $(3,67)$. The relatives of the probands were also recruited when available. Patients with known syndromic CHD or chromosomal anomaly were ruled out from this research. Patients were diagnosed with syndromic CHD if they manifested a distinct facial gestalt or had at least one reported extra-cardiac malformation (85). A total of 400 unrelated volunteers without CHD were enrolled as control individuals from the same geographic area, who were exactly matched with the cases for sex and ethnicity, as well as age. All research participants underwent a comprehensive clinical assessment, as described previously (67-69). This research was fulfilled in compliance with the tenets of the Declaration of Helsinki. The protocol applied to the current investigation was approved by the Medical Ethics Committee of Tongji Hospital, Tongji University School of Medicine [Shanghai, China; approval no. LL(H)-09-07]. Written informed consent was provided by the research participants or their parents prior to commencement of sample collection.

Genetic analysis of KLF13. A whole blood specimen was collected from each study participant in an EDTA-coated tube and stored in a refrigerator at $-80^{\circ} \mathrm{C}$. Genomic DNA was purified from blood leucocytes by utilizing DNA extraction reagent (Promega Corporation). The entire coding region, as well as splicing boundaries, of the KLF13 gene (NC_000015.10) were amplified via polymerase chain reaction (PCR) using a DNA polymerase kit (Qiagen $\mathrm{GmbH}$ ) and the KLF13-specific oligonucleotide primers, as described previously (67): Forward 
5'-CCATGCGCTCACTCTTCGGT-3' and reverse, 5'-CCTTTG TCTGAGGCCGGGCT-3' for the first party of coding exon 1 (product size, $670 \mathrm{bp}$ ); forward, 5'-CGGACCTCAACCAGC AAGCG-3' and reverse, 5'-CTCCGAGAGCCAAGACCCGC-3' for the second party of coding exon 1 (product size, $569 \mathrm{bp}$ ); and forward, 5'-GCATGTGGGAGGGGTGTTGA-3' and reverse, 5'-TCGTGAAACGTGTCCATCCCT-3' for coding exon 2 (product size, $675 \mathrm{bp}$ ). Each mixture used for PCR was prepared in a 0.2-ml PCR tube with a final volume of $25 \mu 1$, containing $1 \mathrm{X}$ Buffer (Qiagen GmbH), 1X Q solution, a component of the HotStar Taq DNA Polymerase kit facilitating amplification of templates with a high-degree secondary structure or with a rich GC content by modifying the melting behavior of DNA (Qiagen $\mathrm{GmbH}), 0.2 \mathrm{mM}$ dNTPs (Qiagen $\mathrm{GmbH}), 0.5 \mu \mathrm{M}$ forward primer, $0.5 \mu \mathrm{M}$ reverse primer, $0.02 \mathrm{U} / \mu 1$ HotStar Taq DNA Polymerase (Qiagen $\mathrm{GmbH}$ ) and $0.1 \mu \mathrm{g}$ genomic DNA. PCR was fulfilled on a 96-well thermocycler (Bio-Rad Laboratories, Inc.). The thermocycling conditions set for the PCR were as follows: Initial denaturation at $95^{\circ} \mathrm{C}$ for $15 \mathrm{~min}$, followed by 36 cycles of denaturation at $94^{\circ} \mathrm{C}$ for $30 \mathrm{sec}$, annealing at $62^{\circ} \mathrm{C}$ for $30 \mathrm{sec}$ and extension at $72^{\circ} \mathrm{C}$ for $1 \mathrm{~min}$, with a final extension at $72^{\circ} \mathrm{C}$ for $7 \mathrm{~min}$. PCR products were resolved by $1.5 \%$ agarose gel electrophoresis and visualized after ethidium bromide staining of gels. PCR-sequencing of extracted amplicons was conducted as described previously (69). For a validated KLF13 variation, the Human Gene Mutation Database (HGMD; http://www.hgmd. cf.ac.uk/ac/index.php), Single Nucleotide Polymorphism (SNP) datbase (https://www.ncbi.nlm.nih.gov/snp) and the Genome Aggregation Database (gnomAD; https://gnomad.broadinstitute.org) were retrieved to verify its novelty.

Construction of gene expression plasmid and site-directed mutagenesis. The wild-type KLF13-pcDNA3.1 plasmid (Invitrogen; Thermo Fisher Scientific, Inc.) was constructed as described previously (67). The mutation discovered in the current study was introduced into wild-type KLF13-pcDNA3.1 by site-targeted mutagenesis with a site-targeted mutagenesis kit (Stratagene; Agilent Technologies Inc.) with the following primers: Forward, 5'-CCCGCGGGGAGCGGCTAGCCCGGC CTCAGAC-3' and reverse, 5'-GTCTGAGGCCGGGCTAGC CGCTCCCCGCGGG-3'. The mutant-type KLF13-pcDNA3.1 was selected by DpnI (Takara Biotechnology Co., Ltd.) and was confirmed by sequencing analysis. The TBX5-pcDNA3.1 plasmid (Invitrogen; Thermo Fisher Scientific, Inc.) and the reporter plasmid of human natriuretic peptide precursor A-luciferase (NPPA-luc), which expresses firefly luciferase, have been described previously (68). The reporter plasmid of human vascular endothelial growth factor A (VEGFA)-luc, which expresses firefly luciferase, was generated as previously described (86).

Cell culture, expression plasmid transfection and reporter gene assay. NIH3T3 cells (Cell Bank of Type Culture Collection of the Chinese Academy of Sciences) were seeded onto a 24-well plate, and maintained in DMEM (Merck KGaA) containing $10 \%$ fetal bovine serum and $1 \%$ penicillin/streptomycin (both Thermo Fisher Scientific, Inc.), in an atmosphere of $5 \% \mathrm{CO}_{2}$ at $37^{\circ} \mathrm{C}$. NIH3T3 cells were transfected $24 \mathrm{~h}$ after plating with various expression plasmids, including empty pcDNA3.1, wild-type KLF13-pcDNA3.1 (KLF13),
Glu144*-mutant KLF13-pcDNA3.1 (Glu144*), wild-type TBX5-pcDNA3.1 (TBX5), NPPA-luc and VEGFA-luc, utilizing the Lipofectamine ${ }^{\circledR} 3000$ Transfection Reagent (Invitrogen; Thermo Fisher Scientific, Inc.) as described previously (67). The internal control plasmid pGL4.75 (Promega Corporation), which expresses Renilla luciferase, was co-transfected to balance transfection efficiency. The empty pcDNA3.1 plasmid (Invitrogen; Thermo Fisher Scientific, Inc.) was used as a negative control. Cells were collected at $48 \mathrm{~h}$ post-transfection, and lysed in $0.2 \mathrm{ml}$ Reporter Lysis Buffer (Promega Corporation). The cellular lysates were used to measure the luciferase activities of firefly and Renilla on a luminometer (Promega Corporation), using a dual-luciferase assay kit (Promega Corporation). The activity of the target gene promoter was expressed as fold activation of firefly luciferase to Renilla luciferase. For each expression plasmid, three transfection experiments were performed in triplicate.

Statistical analysis. Data for promoter activity are presented as the mean \pm standard deviation of the original results from three transfection experiments. Differences in promoter activities between two groups were compared with unpaired Student's t-test. When comparisons among multiple groups were made, one-way ANOVA with a Tukey-Kramer HSD post-hoc test was used. A two-sided P-value of $<0.05$ was used to indicate a statistically significant difference. The statistical software used for the analysis was SPSS version 17.0 for Windows (SPSS, Inc.).

\section{Results}

Clinical characteristic data of the studied patients. In this investigation, a cohort of 316 unrelated index patients suffering from various types of CHD (168 male cases and 148 female cases, with ages ranging from 1-49 years and a mean age of $21 \pm 9$ years) was clinically investigated in contrast to a total of 400 unrelated individuals without CHD (212 male individuals and 188 female individuals, with ages ranging from 1-49 years and a mean age of $21 \pm 8$ years). All the included patients with CHD had echocardiographic evidence, whereas the echocardiograms of the enrolled control subjects were normal, without evidence of cardiovascular structural abnormalities. Among the 316 unrelated index patients with CHD, 58 index patients reported a positive family history of CHD, while all 400 control subjects lacked a family history of CHD. No research participants had known environmental risk factors predisposing them to CHD, including maternal viral infection, nutritional deficiency, obesity, diabetes or exposure to toxic chemicals, therapeutic drugs and ionizing radiation during early pregnancy. Most of the patients underwent cardiac catheterization or surgery. The clinical features of the 316 index cases with CHD are summarized in Table I.

Detection of a new pathogenic KLF13 mutation. Via direct sequencing analysis of the entire coding region and splicing donors/acceptors of KLF13 in 316 index patients with diverse forms of CHD, a heterozygous non-synonymous mutation, NM_015995.3: c.430G $>$ T; p.(Glu144*), was detected in one index patient inflicted with CHD, comprising PDA and VSD, as well as BAV; this mutation therefore has a prevalence of $\sim 0.32 \%$ 
Table I. Demographic and clinical characteristics of the 316 patients affected with various forms of congenital heart disease.

\begin{tabular}{lc}
\hline Parameters & n or mean \\
\hline Sex, n (\%) & \\
Male & $168(53)$ \\
Female & $148(47)$ \\
Mean age (range), years & $21 \pm 9(1-49)$ \\
Distribution of distinct forms of CHD, n (\%) & \\
VSD & $73(23)$ \\
ASD & $57(18)$ \\
PDA & $38(12)$ \\
TOF & $32(10)$ \\
DORV & $16(5)$ \\
VSD + PDA & $28(9)$ \\
VSD + ASD & $22(7)$ \\
DORV + VSD & $16(5)$ \\
TGA + VSD & $16(5)$ \\
ASD + PDA & $9(3)$ \\
TOF + ASD & $6(2)$ \\
PTA + VSD & $3(1)$ \\
Arrhythmias, n (\%) & \\
AVB & $177(56)$ \\
FVPB & $101(32)$ \\
AF & $38(12)$ \\
PVT & $9(3)$ \\
Medical history, n (\%) & $7(2)$ \\
Cardiovascular surgery for CHD & \\
Fatheter-based treatment for CHD & \\
\hline
\end{tabular}

CHD, congenital heart disease; VSD, ventricular septal defect; ASD, atrial septal defect; PDA, patent ductus arteriosus; DORV, double outlet of the right ventricle; TOF, tetralogy of Fallot; TGA, transposition of the great arteries; PTA, persistent truncus arteriosus; AVB, atrioventricular block; FVPB, frequent ventricular premature beat; $\mathrm{AF}$, atrial fibrillation; PVT, paroxysmal ventricular tachycardia.

(1/316) in the study CHD population. The mutation carrier possessed a CHD-positive family history, and genetic assay of the available family members unveiled that the nonsense mutation was in co-segregation with autosomal-dominant CHD in the whole family, with complete penetrance for PDA, as well as BAV, and incomplete penetrance for VSD. The nonsense mutation was neither detected in 400 control subjects nor found in the HGMD, SNP and gnomAD databases, indicating its novelty. The sequence electropherogram traces illustrating the KLF13 mutation in the heterozygous status as well as its wild-type sequence in the homozygous status are presented in Fig. 1A. The schemas exhibiting the functional domains of both wild-type KLF13 and mutant KLF13 are provided in Fig. 1B. The pedigree structure of the studied family inflicted with $\mathrm{CHD}$ is displayed in Fig. 1C. The clinical characteristic information of the CHD-affected family members is shown in Table II.
Transcriptional activation function of KLF13 is disabled by the mutation. As indicated in Fig. 2, wild-type KLF13 and Glu144*-mutant KLF13 (each 100 ng of expression plasmid) transactivated the NPPA promoter by $\sim 16$-fold and $\sim 1$-fold, respectively (wild-type KLF13 vs. Glu144*-mutant KLF13: $\mathrm{t}=9.99854, \mathrm{P}=0.00056)$. When half the amount of wild-type KLF13 and Glu144*-mutant KLF13 (each 50 ng of expression plasmid) was utilized in combination to model the pathological state of mutation carriers (heterozygosity), the induced transcriptional activity was $\sim 8$-fold (wild-type KLF13 vs. wild-type KLF13 + Glu144*-mutant KLF13: $\mathrm{t}=5.31149$, $\mathrm{P}=0.00604)$.

Synergistic transcriptional activation between KLF13 and TBX5 is nullified by the mutation. As indicated in Fig. 3, wild-type KLF13 and Glu144*-mutant KLF13 (each $100 \mathrm{ng}$ of expression plasmid) transactivated the VEGFA promoter by $\sim 9$-fold and $\sim 1$-fold, respectively (wild-type KLF13 vs. Glu144*-mutant KLF13: $\mathrm{t}=9.90028, \mathrm{P}=0.00058)$. In the presence of wild-type TBX5 (100 ng of expression plasmid), wild-type KLF13 and Glu144*-mutant KLF13 (each 100 ng of expression plasmid) transactivated the VEGFA promoter by $\sim 36$-fold and $\sim 4$ fold, respectively (wild-type KLF13 + wild-type TBX5 vs. Glu144*-mutant KLF13 + wild-type TBX5: $\mathrm{t}=16.2934, \mathrm{P}=0.00008)$.

\section{Discussion}

In the present study, a new KLF13 mutation, NM_015995.3: c.430G>T; p.(Glu144*), was identified in one family suffering from PDA, BAV and VSD. The nonsense heterozygous mutation, which co-segregated with CHD in the whole family, was neither observed in 800 control chromosomes nor found in the databases of HGMD, SNP and gnomAD. Functional investigations demonstrated that the Glu144*-mutant KLF13 failed to transcriptionally activate the promoters of NPPA and $V E G F A$. Additionally, the mutation abrogated the synergistic transcriptional activation between KLF13 and TBX5, a well-established CHD-causative gene $(68,69,84)$. These findings support the fact that genetically defective $K L F 13$ confers an enhanced susceptibility to CHD, including PDA, BAV and VSD. Notably, the experiments performed in one NIH3T3 cell line only failed to control for cell-dependent effects, and it remains possible, in fact likely, that other cells may yield different results. Hence, it is very important that additional cells lines are used to examine the functional effect of Glu144*-mutant KLF13 to generalize the mechanism proposed on the basis of this work.

The KLF13 gene, which encodes a transcription factor with 288 amino acids, is located on human chromosome $15 q 13.3$. As one member of the KLF family, the KLF13 protein harbors four critical structural domains encompassing a transactivation domain, which functions to transactivate downstream target genes, a transcriptional inhibition domain, which serves to transcriptionally inhibit downstream target genes, a nuclear localization signals responsible for nuclear localization, and three zinc-fingers required for binding to target gene promoters and interaction with other transcriptionally cooperative partners $(67,87)$. Previous investigations have substantiated the ample expression of KLF13 in the hearts 
A

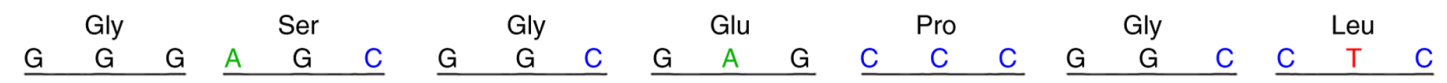

Wild-type
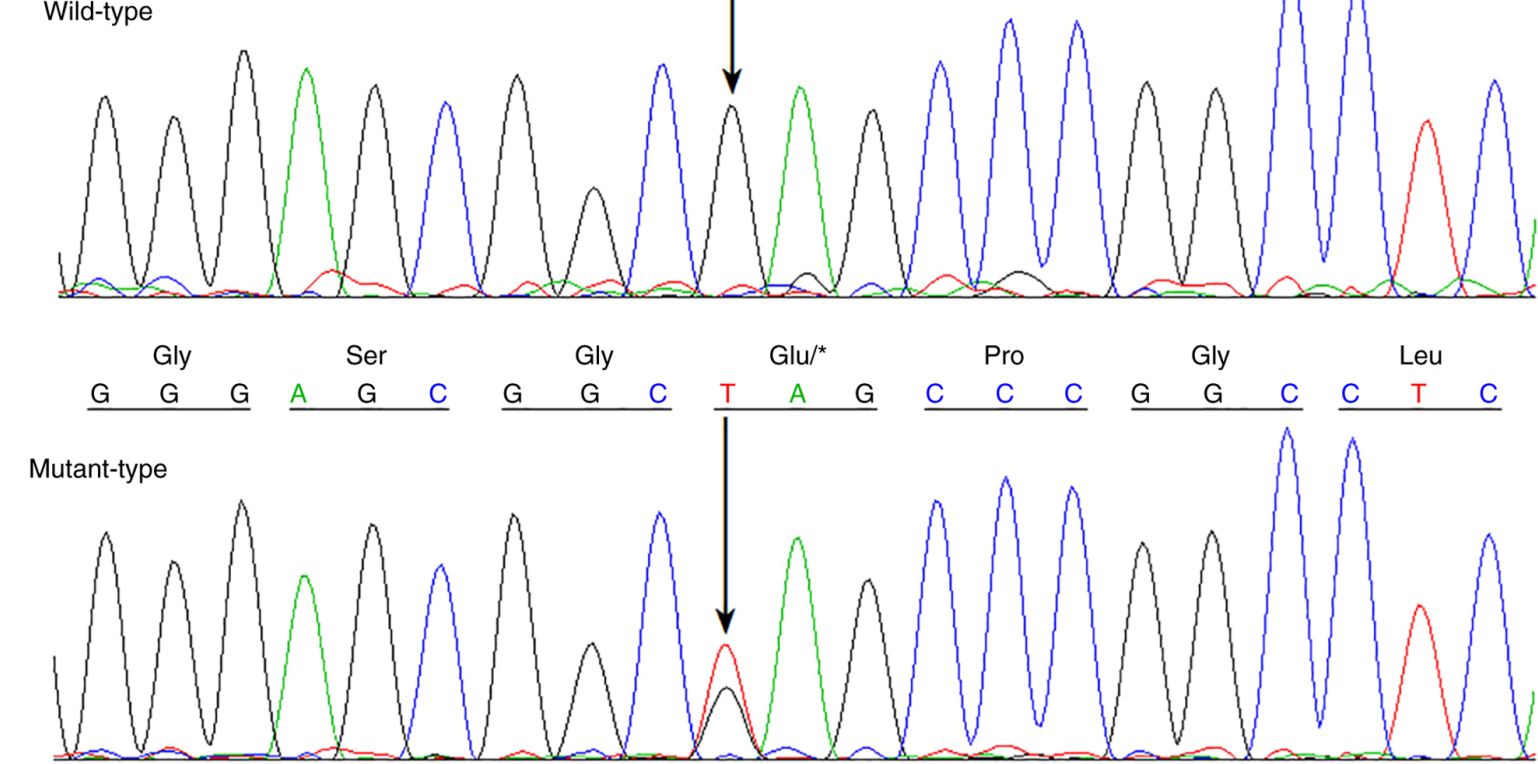

B
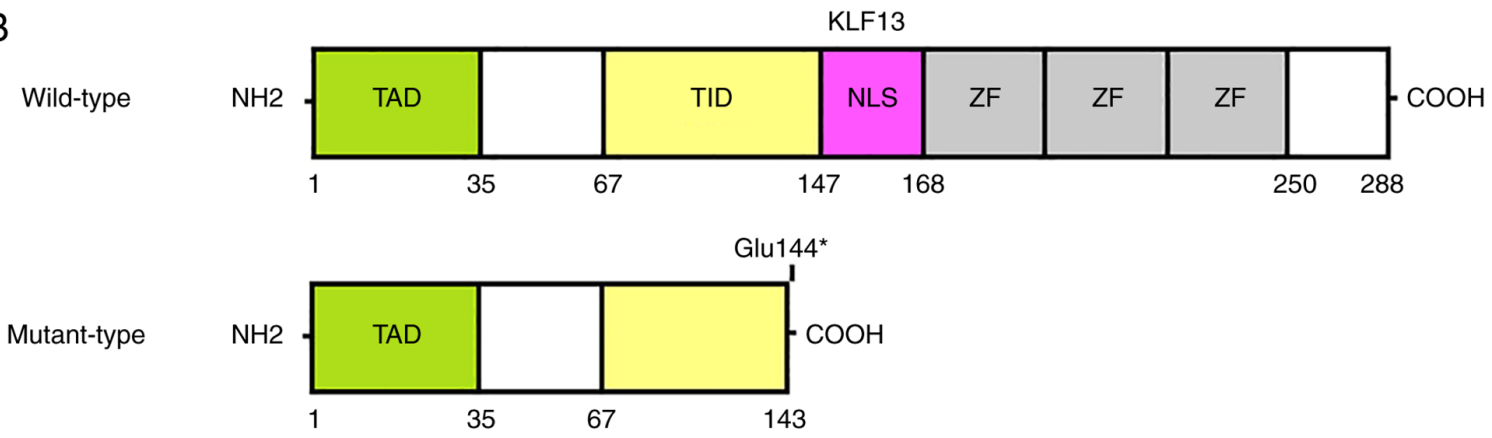

C

Family 1

I

II

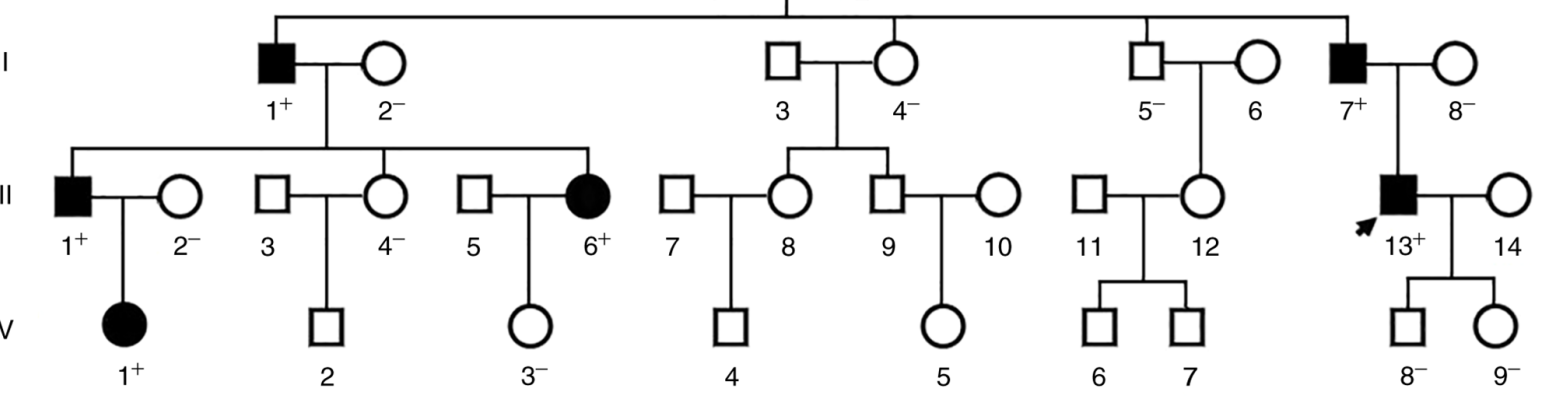

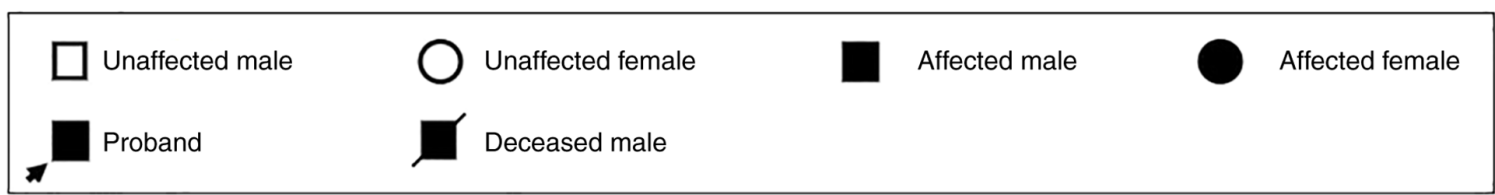

Figure 1. A new KLF13 mutation contributing to congenital heart disease. (A) Sequence electropherograms illustrating the KLF13 mutation in the heterozygous status (mutant) and its wild-type base in the homozygous status (wild-type). The arrow indicates the nucleotides where the mutation occurs. (B) Schematic representations exhibiting the structural domains of KLF13. (C) Pedigree structure of the family inflicted with congenital heart disease (+ represents a carrier of the KLF13 mutation and-represents a non-carrier). TAD, transcriptional activation domain; TID, transcriptional inhibitory domain; NLS, nuclear location signal; $\mathrm{Zn}$, zinc finger.

of both humans and vertebrates during embryonic development, and the pivotal effect of KLF13 on cardiovascular morphogenesis $(66,67,86,88)$. Recent experimental studies have corroborated that KLF13 transactivates the expression 
Table II. Phenotypic profile and KLF13 mutation status of the family members affected with congenital heart disease.

\begin{tabular}{llclr}
\hline Individual & Sex & Age, years & Cardiac phenotype & KLF13 mutation (p.Glu144*) \\
\hline I-1 & M & $75^{\mathrm{a}}$ & PDA, BAV, VSD & NA \\
II-1 & M & 61 & PDA, BAV & $+/-$ \\
II-7 & M & 53 & PDA, BAV, VSD & $+/-$ \\
III-1 & M & 36 & PDA, BAV & $+/-$ \\
III-6 & F & 30 & PDA, BAV & $+/-$ \\
III-13 & M & 28 & PDA, BAV, VSD & $+/-$ \\
IV-1 & F & 10 & PDA, BAV & $+/-$
\end{tabular}

${ }^{\mathrm{a}}$ Age at death. M, male; F, female; PDA, patent ductus arteriosus; BAV, bicuspid aortic valve; VSD, ventricular septal defect; NA, not available; $+/-$, heterozygosity; KLF13, Kruppel-like factor 13.

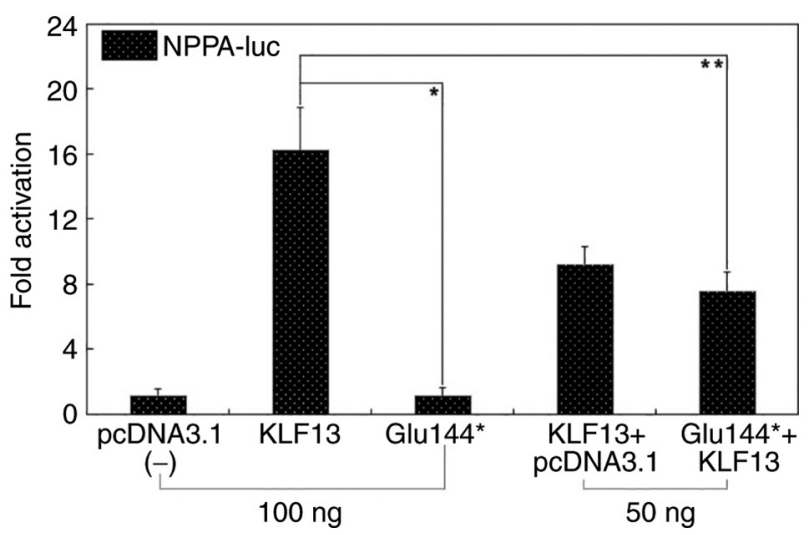

Figure 2. Diminished transcriptional activity of KLF13 caused by the mutation. In cultivated NIH3T3 cells expressing various recombinant plasmids, activation of NPPA-luc by wild-type or Glu144*-mutant KLF13, singly or together, revealed that Glu144*-mutant KLF13 failed to transactivate its downstream target gene NPPA. For each eukaryotic expression plasmid, cellular transient transfection experiments were repeated three times in triplicates. ${ }^{*} \mathrm{P}=0.00056$ and ${ }^{* *} \mathrm{P}=0.00604$ compared with wild-type KLF13. NPPA-luc, human natriuretic peptide precursor A luciferase; KLF13, Kruppel-like factor 13.

of several downstream genes, encompassing $N P P A, N P P B$, $V E G F A$ and $A C T C 1$, separately or synergistically with TBX5, GATA4 and GATA6 $(86,88)$. Deleterious mutations in KLF13 and its downstream target genes VEGFA and ACTC1, as well as its transcriptionally cooperative partners TBX5, GATA4 and GATA6, have been discovered as genetic defects underpinning CHD in humans (66-69,84,89-91). In the current investigation, the mutation found in cases with familial CHD was anticipated to generate a truncated KLF13 protein losing most structural domains, and biochemical assays revealed that Glu144*-mutant KLF13 had no transactivation on its downstream target genes. In addition, the mutation disrupted the synergistic transcriptional activation between KLF13 and TBX5. These results strongly indicate $K L F 13$ haploinsufficiency as a molecular mechanism underpinning CHD in a subset of cases.

It has been validated in experimental animal models that Klf13 deficiency contributes to aberrant cardiovascular morphogenesis. In Xenopus, KLF13 is abundantly expressed in the heart and vessels during embryogenesis, and knockdown of the Klf13 alleles in Xenopus embryos leads to atrial

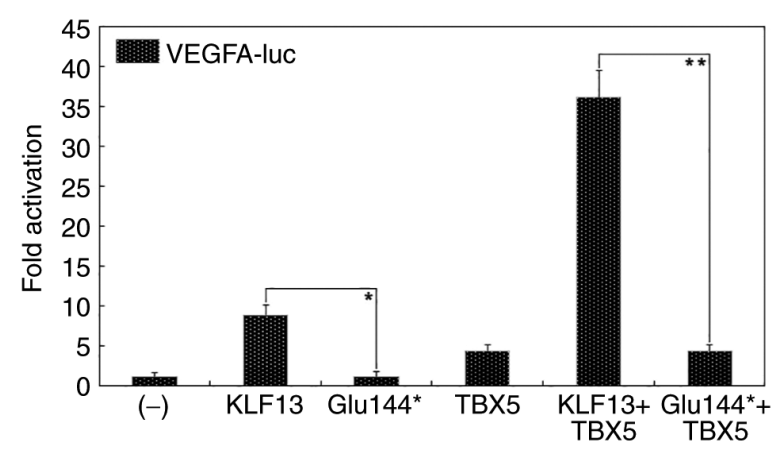

Figure 3. Synergistic transcriptional activation between KLF13 and TBX5 disrupted by the mutation. In cultivated NIH3T3 cells, the synergistic transactivation of the promoter of VEGFA by KLF13 and TBX5 was abrogated by the Glu144* mutation. ${ }^{*} \mathrm{P}=0.00058$ and ${ }^{* *} \mathrm{P}=0.00008$ compared with to respective wild-type counterparts. VEGFA, human vascular endothelial growth factor A luciferase; TBX5, T-box transcription factor 5; KLF13, Kruppel-like factor 13.

septal defects and myocardial trabecular hypoplasia, similar to those seen in mice or humans with hypomorphic alleles of Gata4 (88). In mice, KLF13 is expressed highly in the heart at all stages of embryonic development, including during development of truncus arteriosus, ventricular trabeculae, atrial myocardium and atrioventricular cushions $(88,92)$. When homozygous knockout of Klf13 alleles was performed in mice, cardiac vacuolar lesions, heart enlargement and embryonic death occurred (93). In another study, although mice with heterozygous deletion of Klf13 had no obvious cardiac structural abnormalities, compound haploinsufficiency of Tbx5 and Klf13 markedly reduced the postnatal viability and significantly increased the penetrance of cardiac septal aberrations caused by $T b x 5$ haploinsufficiency (88). Altogether, these results from experimental animals indicate that genetically defective $K L F 13$ increases the vulnerability to CHD in humans, and suggest that the dosage of KLF13 must be finely controlled to avoid cardiovascular developmental malformations.

Notably, KLF13 variations have been reported to cause distinct types of congenital cardiovascular deformities in humans, including TGA, TVA, DORV, VSD and ASD $(66,67)$. In the present study, the affected family members who carried an identified KLF13 mutation manifested PDA, BAV and VSD, therefore expanding the phenotypic spectrum ascribed to mutant $K L F 13$ and highlighting the genetic heterogeneity of CHD. 
In conclusion, the present study causally links a new $K L F 13$ mutation to $\mathrm{CHD}$, and to the best of our knowledge, for the first time to PDA and BAV, which reveals a novel molecular pathogenesis underlying $\mathrm{CHD}$, conducive to precise prophylaxis and personalized treatment of the affected patients.

\section{Acknowledgements}

Not applicable.

\section{Funding}

This study received financial support from the Basic Research Project of Shanghai, China (grant no. 20JC1418800), the Natural Science Foundation of Shanghai, China (grant no. 18ZR1431000) and the Natural Science Foundation of Minhang District, Shanghai, China (grant no. 2020MHZ083).

\section{Availability of data and materials}

The datasets used and/or analyzed during the current study are available from the corresponding author on reasonable request.

\section{Authors' contributions}

JW and YQY designed the investigation, and made leading contributions to the writing of manuscript. PA, GFZ, CMZ, YJX, JW and YQY completed the clinical investigations. PA, GFZ, JW and YQY fulfilled the genetic and biochemical studies. JW and YQY confirm the authenticity of all the raw data. All authors have read and approved the final version of manuscript.

\section{Ethics approval and consent to participate}

This investigation was approved by the Medical Ethics Committee of Tongji Hospital, Tongji University School of Medicine [Shanghai, China; approval no. LL(H)-09-07]. Written informed consent was collected from the study individuals or their parents prior to investigation.

\section{Patient consent for publication}

Not applicable.

\section{Competing interests}

The authors declare that they have no competing interests.

\section{References}

1. Diab NS, Barish S, Dong W, Zhao S, Allington G, Yu X, Kahle KT, Brueckner M and Jin SC: Molecular genetics and complex inheritance of congenital heart disease. Genes (Basel) 12: 1020, 2021.

2. Martin LJ and Benson DW: Focused strategies for defining the genetic architecture of congenital heart defects. Genes (Basel) 12: 827, 2021.

3. Benjamin EJ, Muntner P, Alonso A, Bittencourt MS, Callaway CW, Carson AP, Chamberlain AM, Chang AR, Cheng S, Das SR, et al: Heart disease and stroke statistics-2019 update: A report from the American Heart Association. Circulation 139: e56-e528, 2019.
4. Skeffington KL, Bond AR, Bigotti MG, AbdulGhani S, Iacobazzi D, Kang SL, Heesom KJ, Wilson MC, Stoica S, Martin R, et al: Changes in inflammation and oxidative stress signalling pathways in coarcted aorta triggered by bicuspid aortic valve and growth in young children. Exp Ther Med 20: 48, 2020.

5. Dragomir C, Manea AM, Enatescu VR, Lacatusu AAM, Lacatusu A, Henry OI, Boia M and Ilie C: Left heart hypoplasia operated using double pulmonary arterial banding with double arterial duct stenting: A case report. Exp Ther Med 20: 193, 2020.

6. Hu C, Huang S, Wu F and Ding H: MicroRNA-219-5p participates in cyanotic congenital heart disease progression by regulating cardiomyocyte apoptosis. Exp Ther Med 21: 36, 2021.

7. Andonian CS, Freilinger S, Achenbach S, Ewert $P$, Gundlach U, Hoerer J, Kaemmerer H, Pieper L, Weyand M, Neidenbach RC, et al: 'Well-being paradox' revisited: A cross-sectional study of quality of life in over 4000 adults with congenital heart disease. BMJ Open 11: e049531, 2021.

8. Brudy L, Meyer M, Oberhoffer R, Ewert P and Müller J: Move more-be happier? physical activity and health-related quality of life in children with congenital heart disease. Am Heart J 241: 68-73, 2021.

9. Moons P, Luyckx K, Thomet C, Budts W, Enomoto J, Sluman MA, Lu CW, Jackson JL, Khairy P, Cook SC, et al: Physical functioning, mental health, and quality of life in different congenital heart defects: Comparative analysis in 3538 patients from 15 countries. Can J Cardiol 37: 215-223, 2021.

10. Hayama Y, Ohuchi H, Negishi J, Iwasa T, Sakaguchi H, Miyazaki A, Tsuda E and Kurosaki K: Effect of stiffened and dilated ascending aorta on aerobic exercise capacity in repaired patients with complex congenital heart disease. Am J Cardiol 129: 87-94, 2020.

11. Spiesshoefer J, Orwat S, Henke C, Kabitz HJ, Katsianos S, Borrelli C, Baumgartner H, Nofer JR, Spieker M, Bengel P, et al: Inspiratory muscle dysfunction and restrictive lung function impairment in congenital heart disease: Association with immune inflammatory response and exercise intolerance. Int J Cardiol 318: 45-51, 2020.

12. Meyer M, Brudy L, García-Cuenllas L, Hager A, Ewert P, Oberhoffer R and Müller J: Current state of home-based exercise interventions in patients with congenital heart disease: A systematic review. Heart 106: 333-341, 2020.

13. Xu C, Su X, Ma S, Shu Y, Zhang Y, Hu Y and Mo X: Effects of exercise training in postoperative patients with congenital heart disease: A systematic review and meta-analysis of randomized controlled trials. J Am Heart Assoc 9: e013516, 2020.

14. MeyerM,Brudy L,Fuertes-Moure A,Hager A,Oberhoffer-Fritz R, Ewert $\mathrm{P}$ and Müller J: E-health exercise intervention for pediatric patients with congenital heart disease: A randomized controlled trial. J Pediatr 233: 163-168, 2021.

15. Asschenfeldt B, Evald L, Heiberg J, Salvig C, Østergaard L, Dalby RB, Eskildsen SF and Hjortdal VE: Neuropsychological status and structural brain imaging in adults with simple congenital heart defects closed in childhood. J Am Heart Assoc 9: $\mathrm{e} 015843,2020$.

16. Kessler N, Feldmann M, Schlosser L, Rometsch S, Brugger P, Kottke R, Knirsch W, Oxenius A, Greutmann M and Latal B: Structural brain abnormalities in adults with congenital heart disease: Prevalence and association with estimated intelligence quotient. Int J Cardiol 306: 61-66, 2020.

17. Bonthrone AF, Dimitrova R, Chew A, Kelly CJ Cordero-Grande L, Carney O, Egloff A, Hughes E, Vecchiato K, Simpson J, et al: Individualized brain development and cognitive outcome in infants with congenital heart disease. Brain Commun 3: fcab046, 2021.

18. Gui J, Liang S, Sun Y, Liu Y, Chen C, Wang B, Zhong J, Yu Y and He S: Effect of perioperative amplitude-integrated electroencephalography on neurodevelopmental outcomes following infant heart surgery. Exp Ther Med 20: 2879-2887, 2020.

19. Giang KW, Mandalenakis Z, Dellborg M, Lappas G, Eriksson P, Hansson PO and Rosengren A: Long-term risk of hemorrhagic stroke in young patients with congenital heart disease. Stroke 49: 1155-1162, 2018.

20. Giang KW, Fedchenko M, Dellborg M, Eriksson $P$ and Mandalenakis Z: Burden of ischemic stroke in patients with congenital heart disease: a nationwide, case-control study. J Am Heart Assoc 10: e020939, 2021.

21. Freisinger E, Gerß J, Makowski L, Marschall U, Reinecke H, Baumgartner H, Koeppe J and Diller GP: Current use and safety of novel oral anticoagulants in adults with congenital heart disease: Results of a nationwide analysis including more than 44000 patients. Eur Heart J 41: 4168-4177, 2020. 
22. Diller GP, Körten MA, Bauer UM, Miera O, Tutarel O, Kaemmerer H, Berger $F$ and Baumgartner H; German Competence Network for Congenital Heart Defects Investigators: Current therapy and outcome of Eisenmenger syndrome: Data of the German National Register for congenital heart defects. Eur Heart J 37: 1449-1455, 2016.

23. Kaemmerer H, Gorenflo M, Huscher D, Pittrow D, Apitz C, Baumgartner H, Berger F, Bruch L, Brunnemer E, Budts W, et al: Pulmonary hypertension in adults with congenital heart disease: Real-world data from the International COMPERA-CHD Registry. J Clin Med 9: 1456, 2020.

24. Long L, Xiao Y, Yin X, Gao S, Zhou L and Liu H: Expression of serum miR-27b and miR-451 in patients with congenital heart disease associated pulmonary artery hypertension and risk factor analysis. Exp Ther Med 20: 3196-3202, 2020.

25. Diller GP, Enders D, Lammers AE, Orwat S, Schmidt R, Radke RM, Gerss J, De Torres Alba F, Kaleschke G, Bauer UM, et al: Mortality and morbidity in patients with congenital heart disease hospitalised for viral pneumonia. Heart 107: 1069-1076, 2020.

26. Radke RM, Frenzel T, Baumgartner H and Diller GP: Adult congenital heart disease and the COVID-19 pandemic. Heart 106: 1302-1309, 2020.

27. Diller GP, Gatzoulis MA, Broberg CS, Aboulhosn J, Brida M, Schwerzmann M, Chessa M, Kovacs AH and Roos-Hesselink J: Coronavirus disease 2019 in adults with congenital heart disease: A position paper from the ESC working group of adult congenital heart disease, and the International Society for Adult Congenital Heart Disease. Eur Heart J 42: 1858-1865, 2021.

28. Diller GP and Baumgartner H: Endocarditis in adults with congenital heart disease: New answers-new questions. Eur Heart J 38: 2057-2059, 2017.

29. Tutarel O, Alonso-Gonzalez R, Montanaro C, Schiff R, Uribarri A, Kempny A, Grübler MR, Uebing A, Swan L, Diller GP, et al: Infective endocarditis in adults with congenital heart disease remains a lethal disease. Heart 104: 161-165, 2018.

30. Cahill TJ, Jewell PD, Denne L, Franklin RC, Frigiola A, Orchard E and Prendergast BD: Contemporary epidemiology of infective endocarditis in patients with congenital heart disease: A UK prospective study. Am Heart J 215: 70-77, 2019.

31. Fedchenko M, Mandalenakis Z, Giang KW, Rosengren A, Eriksson $\mathrm{P}$ and Dellborg $\mathrm{M}$ : Long-term outcomes after myocardial infarction in middle-aged and older patients with congenital heart disease-a nationwide study. Eur Heart J 42: $2577-2586,2021$.

32. Orwat S and Diller GP: Congenital heart defects as an intrinsic additional risk factor for the occurrence and outcome of myocardial infarction. Eur Heart J 42: 2587-2589, 2021.

33. Hirono K, Hata Y, Miyao N, Okabe M, Takarada S, Nakaoka H, Ibuki K, Ozawa S, Yoshimura N, Nishida N, et al: Left ventricular noncompaction and congenital heart disease increases the risk of congestive heart failure. J Clin Med 9: 785, 2020.

34. Menachem JN, Schlendorf KH, Mazurek JA, Bichell DP, Brinkley DM, Frischhertz BP, Mettler BA, Shah AS Zalawadiya S, Book W, et al: Advanced heart failure in adults with congenital heart disease. JACC Heart Fail 8: 87-99, 2020.

35. Arnaert S, De Meester P, Troost E, Droogne W, Van Aelst L, Van Cleemput J, Voros G, Gewillig M, Cools B, Moons P, et al: Heart failure related to adult congenital heart disease: Prevalence, outcome and risk factors. ESC Heart Fail 8: 2940-2950, 2021.

36. SakhiR,Kauling RM, TheunsDA, Szili-TorokT,BhagwandienRE, van den Bosch AE, Cuypers JAAE, Roos-Hesselink JW and Yap SC: Early detection of ventricular arrhythmias in adults with congenital heart disease using an insertable cardiac monitor (EDVA-CHD study). Int J Cardiol 305: 63-69, 2020.

37. Casteigt B, Samuel M, Laplante L, Shohoudi A, Apers S, Kovacs AH, Luyckx K, Thomet C, Budts W, Enomoto J, et al: Atrial arrhythmias and patient-reported outcomes in adults with congenital heart disease: An international study. Heart Rhythm 18: 793-800, 2021.

38. Wasmer K, Eckardt L, Baumgartner H and Köbe J: Therapy of supraventricular and ventricular arrhythmias in adults with congenital heart disease-narrative review. Cardiovasc Diagn Ther 11: 550-562, 2021.

39. Goldstein SA, D'Ottavio A, Spears T, Chiswell K, Hartman RJ, Krasuski RA, Kemper AR, Meyer RE, Hoffman TM, Walsh MJ, et al: Causes of death and cardiovascular comorbidities in adults with congenital heart disease. J Am Heart Assoc 9: e016400, 2020.
40. Oliver JM, Gallego P, Gonzalez AE, Avila P, Alonso A Garcia-Hamilton D, Peinado R, Dos-Subirà L, Pijuan-Domenech A, Rueda J, et al: Predicting sudden cardiac death in adults with congenital heart disease. Heart 107: 67-75, 2021.

41. Vehmeijer JT, Koyak Z, Leerink JM, Zwinderman AH, Harris L, Peinado R, Oechslin EN, Robbers-Visser D, Groenink M, Boekholdt SM, et al: Identification of patients at risk of sudden cardiac death in congenital heart disease: the PRospEctiVE study on implaNTable cardIOverter defibrillator therapy and suddeN cardiac death in Adults with Congenital Heart Disease (PREVENTION-ACHD). Heart Rhythm 18: 785-792, 2021.

42. Williams JL, Torok RD, D'Ottavio A, Spears T, Chiswell K, Forestieri NE, Sang CJ, Paolillo JA, Walsh MJ,Hoffman TM, et al: Causes of death in infants and children with congenital heart disease. Pediatr Cardiol 42: 1308-1315, 2021.

43. Virani SS, Alonso A, Aparicio HJ, Benjamin EJ, Bittencourt MS, Callaway CW, Carson AP, Chamberlain AM, Cheng S, Delling FN, et al: Heart disease and stroke statistics-2021 update: A report from the American Heart Association. Circulation 143: e254-e743, 2021.

44. Diller GP, Arvanitaki A, Opotowsky AR, Jenkins K, Moons P, Kempny A, Tandon A, Redington A, Khairy P, Mital S, et al: Lifespan perspective on congenital heart disease research: JACC state-of-the-art review. J Am Coll Cardiol 77: 2219-2235, 2021.

45. Bouma BJ and Mulder BJ: Changing landscape of congenital heart disease. Circ Res 120: 908-922, 2017.

46. Spector LG, Menk JS, Knight JH, McCracken C, Thomas AS, Vinocur JM, Oster ME, St Louis JD, Moller JH and Kochilas L: Trends in long-term mortality after congenital heart surgery. J Am Coll Cardiol 71: 2434-2446, 2018.

47. Niwa K, Kaemmerer H and von Kodolitsch Y: Current diagnosis and management of late complications in adult congenital heart disease. Cardiovasc Diagn Ther 11: 478-480, 2021.

48. Kalisch-Smith JI, Ved N and Sparrow DB: Environmental risk factors for congenital heart disease. Cold Spring Harb Perspect Biol 12: a037234, 2020.

49. Zhou J, Xiong Y, Dong X, Wang H, Qian Y, Ma D and Li X: Genome-wide methylation analysis reveals differentially methylated $\mathrm{CpG}$ sites and altered expression of heart development-associated genes in fetuses with cardiac defects. Exp Ther Med 22: 1032, 2021.

50. Bigras JL: Cardiovascular risk factors in patients with congenital heart disease. Can J Cardiol 36: 1458-1466, 2020.

51. Helle E and Priest JR: Maternal obesity and diabetes mellitus as risk factors for congenital heart disease in the offspring. J Am Heart Assoc 9: e011541, 2020.

52. Saliba A, Figueiredo AC, Baroneza JE, Afiune JY, Pic-Taylor A, Oliveira SF and Mazzeu JF: Genetic and genomics in congenital heart disease: A clinical review. J Pediatr (Rio J) 96: 279-288, 2020.

53. Shabana NA, Shahid SU and Irfan U: Genetic contribution to congenital heart disease (CHD). Pediatr Cardiol 41: 12-23, 2020.

54. Majumdar U, Yasuhara J and Garg V: In vivo and in vitro genetic models of congenital heart disease. Cold Spring Harb Perspect Biol 13: a036764, 2021.

55. Loffredo CA, Chokkalingam A, Sill AM, Boughman JA, Clark EB, Scheel J and Brenner JI: Prevalence of congenital cardiovascular malformations among relatives of infants with hypoplastic left heart, coarctation of the aorta, and d-transposition of the great arteries. Am J Med Genet A 124A: 225-230, 2004.

56. Arya P, Wilson TE, Parent JJ, Ware SM, Breman AM and Helm BM: An adult female with 5q34-q35.2 deletion: A rare syndromic presentation of left ventricular non-compaction and congenital heart disease. Eur J Med Genet 63: 103797, 2020.

57. Evangelidou P, Kousoulidou L, Salameh N, Alexandrou A, Papaevripidou I, Alexandrou IM, Ketoni A, Ioannidou C, Christophidou-Anastasiadou V, Tanteles GA, et al: An unusual combination of an atypical maternally inherited novel $0.3 \mathrm{Mb}$ deletion in Williams-Beuren region and a de novo 22q11.21 microduplication in an infant with supravalvular aortic stenosis. Eur J Med Genet 63: 104084, 2020.

58. Szot JO, Campagnolo C, Cao Y, Iyer KR, Cuny H, Drysdale T, Flores-Daboub JA, Bi W, Westerfield L, Liu P, et al: Bi-allelic mutations in NADSYN1 cause multiple organ defects and expand the genotypic spectrum of congenital NAD deficiency disorders. Am J Hum Genet 106: 129-136, 2020

59. Chen CA, Crutcher E, Gill H, Nelson TN, Robak LA, Jongmans MC, Pfundt R, Prasad C, Berard RA, Fannemel M, et al: The expanding clinical phenotype of germline ABL1-associated congenital heart defects and skeletal malformations syndrome. Hum Mutat 41: 1738-1744, 2020 
60. Hsieh A, Morton SU, Willcox JAL, Gorham JM, Tai AC, Qi H, DePalma S, McKean D, Griffin E, Manheimer KB, et al: EM-mosaic detects mosaic point mutations that contribute to congenital heart disease. Genome Med 12: 42, 2020.

61. Kolomenski JE, Delea M, Simonetti L, Fabbro MC, Espeche LD, Taboas M, Nadra AD, Bruque CD and Dain L: An update on genetic variants of the NKX2-5. Hum Mutat 41: 1187-1208, 2020

62. Liu H, Giguet-Valard AG, Simonet T, Szenker-Ravi E, Lambert L, Vincent-Delorme C, Scheidecker S, Fradin M, Morice-Picard F, Naudion S, et al: Next-generation sequencing in a series of 80 fetuses with complex cardiac malformations and/or heterotaxy. Hum Mutat 41: 2167-2178, 2020.

63. Lin JI, Feinstein TN, Jha A, McCleary JT, Xu J, Arrigo AB, Rong G, Maclay LM, Ridge T, Xu X, et al: Mutation of LRP1 in cardiac neural crest cells causes congenital heart defects by perturbing outflow lengthening. Commun Biol 3: 312, 2020.

64. Sutani A, Shima H, Hijikata A, Hosokawa S, Katoh-Fukui Y Takasawa K, Suzuki E, Doi S, Shirai T, Morio T, et al: WDR11 is another causative gene for coloboma, cardiac anomaly and growth retardation in 10q26 deletion syndrome. Eur J Med Genet 63: 103626, 2020.

65. Le Fevre A, Baptista J, Ellard S, Overton T, Oliver A, Gradhand E and Scurr I: Compound heterozygous Pkd111 variants in a family with two fetuses affected by heterotaxy and complex Chd. Eur J Med Genet 63: 103657, 2020 .

66. Li W, Li B, Li T, Zhang E, Wang Q, Chen S and Sun K: Identification and analysis of KLF13 variants in patients with congenital heart disease. BMC Med Genet 21: 78, 2020.

67. Wang SS, Wang TM, Qiao XH, Huang RT, Xue S, Dong BB, $\mathrm{Xu}$ YJ, Liu XY and Yang YQ: KLF13 loss-of-function variation contributes to familial congenital heart defects. Eur Rev Med Pharmacol Sci 24: 11273-11285, 2020.

68. Zhang Y, Sun YM, Xu YJ, Zhao CM, Yuan F, Guo XJ, Guo YH, Yang CX, Gu JN, Qiao Q, et al: A new TBX5 loss-of-function mutation contributes to congenital heart defect and atrioventricular block. Int Heart J 61: 761-768, 2020.

69. Jiang WF, Xu YJ, Zhao CM, Wang XH, Qiu XB, Liu X, Wu SH and Yang YQ: A novel TBX5 mutation predisposes to familial cardiac septal defects and atrial fibrillation as well as bicuspid aortic valve. Genet Mol Biol 43: e20200142, 2020

70. Wang C, Lv H, Ling X, Li H, Diao F, Dai J, Du J, Chen T, Xi Q, Zhao Y, et al: Association of assisted reproductive technology, germline de novo mutations and congenital heart defects in a prospective birth cohort study. Cell Res 31: 919-928, 2021.

71. Lahrouchi N, Postma AV, Salazar CM, De Laughter DM, Tjong F, Piherová L, Bowling FZ, Zimmerman D, Lodder EM, Ta-Shma A, et al: Biallelic loss-of-function variants in PLD1 cause congenital right-sided cardiac valve defects and neonatal cardiomyopathy. J Clin Invest 131: e142148, 2021.

72. Audain E, Wilsdon A, Breckpot J, Izarzugaza JM, Fitzgerald TW, Kahlert AK, Sifrim A, Wünnemann F, Perez-Riverol Y, Abdul-Khaliq $\mathrm{H}$, et al: Integrative analysis of genomic variants reveals new associations of candidate haploinsufficient genes with congenital heart disease. PLoS Genet 17: e1009679, 2021.

73. Zheng SQ, Chen HX, Liu XC, Yang Q and He GW: Genetic analysis of the CITED2 gene promoter in isolated and sporadic congenital ventricular septal defects. J Cell Mol Med 25 2254-2261, 2021

74. Fu F, Li R, Lei TY, Wang D, Yang X, Han J, Pan M, Zhen L, Li J, Li FT, et al: Compound heterozygous mutation of the ASXL3 gene causes autosomal recessive congenital heart disease. Hum Genet 140: 333-348, 2021.

75. Hou C, Zheng J, Liu W, Xie L, Sun X, Zhang Y, Xu M, Li Y and Xiao T: Identification and characterization of a novel ELN mutation in congenital heart disease with pulmonary artery stenosis. Sci Rep 11: 14154, 2021.

76. Helm BM, Landis BJ and Ware SM: Genetic evaluation of inpatient neonatal and infantile congenital heart defects: New findings and review of the literature. Genes (Basel) 12: 1244, 2021.

77. Massadeh S, Albeladi M, Albesher N, Alhabshan F, Kampe KD Chaikhouni F, Kabbani MS, Beetz C and Alaamery M: Novel autosomal recessive splice-altering variant in PRKD1 is associated with congenital heart disease. Genes (Basel) 12: 612, 2021.
78. Musfee FI, Agopian AJ, Goldmuntz E, Hakonarson $\mathrm{H}$, Morrow BE, Taylor DM, Tristani-Firouzi M, Watkins WS, Yandell $\mathrm{M}$ and Mitchell LE: Common variation in cytoskeletal genes is associated with conotruncal heart defects. Genes (Basel) 12: 655, 2021.

79. Meerschaut I, Vergult S, Dheedene A, Menten B, De Groote K, De Wilde H, Muiño Mosquera L, Panzer J, Vandekerckhove K, Coucke PJ, et al: A reassessment of copy number variations in congenital heart defects: Picturing the whole genome. Genes (Basel) 12: 1048, 2021.

80. Yadav ML, Jain D, Neelabh, Agrawal D, Kumar A and Mohapatra B: A gain-of-function mutation in CITED2 is associated with congenital heart disease. Mutat Res 822: 111741, 2021.

81. Basel-Salmon L, Ruhrman-Shahar N, Barel O, Hagari O, Marek-Yagel D, Azulai N, Bazak L, Svirsky R, Reznik-Wolf H, Lidzbarsky GA, et al: Biallelic variants in ETV2 in a family with congenital heart defects, vertebral abnormalities and preaxial polydactyly. Eur J Med Genet 64: 104124, 2021.

82. Huang S, Wu Y, Chen S, Yang Y, Wang Y, Wang H, Li P, Zhuang J and Xia Y: Novel insertion mutation (Arg1822 Glu1823dup) in MYH6 coiled-coil domain causing familial atrial septal defect. Eur J Med Genet 64: 104314, 2021.

83. Zhao L, Jiang WF, Yang CX, Qiao Q, Xu YJ, Shi HY, Qiu XB, Wu SH and Yang YQ: SOX17 loss-of-function variation underlying familial congenital heart disease. Eur J Med Genet 64: 104211, 2021.

84. Li YJ and Yang YQ: An update on the molecular diagnosis of congenital heart disease: Focus on loss-of-function mutations. Expert Rev Mol Diagn 17: 393-401, 2017.

85. Sifrim A, Hitz MP, Wilsdon A, Breckpot J, Turki SH, Thienpont B, McRae J, Fitzgerald TW, Singh T, Swaminathan GJ, et al: Distinct genetic architectures for syndromic and nonsyndromic congenital heart defects identified by exome sequencing. Nat Genet 48: 1060-1065, 2016.

86. Darwich R, Li W, Yamak A, Komati H, Andelfinger G, Sun K and Nemer M: KLF13 is a genetic modifier of the Holt-Oram syndrome gene TBX5. Hum Mol Genet 26: 942-954, 2017.

87. Song A, Patel A, Thamatrakoln K, Liu C, Feng D, Clayberger C and Krensky AM: Functional domains and DNA-binding sequences of RFLAT-1/KLF13, a Krüppel-like transcription factor of activated T lymphocytes. J Biol Chem 277: 30055-30065, 2002.

88. Lavallée G, Andelfinger G, Nadeau M, Lefebvre C, Nemer G, Horb ME and Nemer M: The Kruppel-like transcription factor KLF13 is a novel regulator of heart development. EMBO J 25: 5201-5213, 2006

89. Zhao W, Wang J, Shen J, Sun K, Zhu J, Yu T, Ji W, Chen Y, Fu Q and Li F: Mutations in VEGFA are associated with congenital left ventricular outflow tract obstruction. Biochem Biophys Res Commun 396: 483-488, 2010

90. Greenway SC, McLeod R, Hume S, Roslin NM, Alvarez N, Giuffre M, Zhan SH, Shen Y, Preuss C, Andelfinger G, et al: Exome sequencing identifies a novel variant in ACTC1 associated with familial atrial septal defect. Can J Cardiol 30: 181-187, 2014.

91. Matsson H, Eason J, Bookwalter CS, Klar J, Gustavsson P, Sunnegårdh J, Enell H, Jonzon A, Vikkula M, Gutierrez I, et al: Alpha-cardiac actin mutations produce atrial septal defects. Hum Mol Genet 17: 256-265, 2008.

92. Martin KM, Metcalfe JC and Kemp PR: Expression of Klf9 and Klf13 in mouse development. Mech Dev 103: 149-151, 2001.

93. Gordon AR, Outram SV, Keramatipour M, Goddard CA, Colledge WH, Metcalfe JC, Hager-Theodorides AL, Crompton T and Kemp PR: Splenomegaly and modified erythropoiesis in KLF13 $^{-/}$mice. J Biol Chem 283: 11897-11904, 2008.

This work is licensed under a Creative Commons Attribution-NonCommercial-NoDerivatives 4.0 International (CC BY-NC-ND 4.0) License. 